\title{
Prognosis of Mild Cognitive Impairment in General Practice: Results of the German AgeCoDe Study
}

\author{
Hanna Kaduszkiewicz, MD, Prof* \\ Marion Eisele, $P b D^{*}$ \\ Birgitt Wiese, MSc \\ Jana Prokein, MSc \\ Melanie Luppa, PbD \\ Tobias Luck, $P b D$ \\ Frank Jessen, MD, Prof \\ Horst Bickel, PbD \\ Edelgard Mösch, $P b D$ \\ Michael Pentzek, PbD \\ Angela Fuchs, MSc \\ Sandra Eifflaender-Gorfer, PbD \\ Siegfried Weyerer, PbD, Prof \\ Hans-Helmut König, MD, MPH, Prof \\ Christian Brettschneider, MSc \\ Hendrik van den Bussche, PbD, Prof \\ Wolfgang Maier, MD, Prof \\ Martin Scherer, MD, Prof \\ Steffi G. Riedel-Heller, MD, Prof \\ for the Study on Aging, Cognition, \\ and Dementia in Primary Care \\ Patients (AgeCoDe) Study Group \\ *Shared first authors.
}

Conflicts of interest: authors report none.

\section{CORRESPONDING AUTHORS}

Marion Eisele, $\mathrm{PhD}$

University Medical Center,

Hamburg-Eppendorf

Department of Primary Medical Care

Center for Psychosocial Medicine

Martinistraße 52

D-20246 Hamburg, Germany

m.eisele@uke.uni-hamburg.de

Hanna Kaduszkiewicz, MD, Prof

University Medical Center

Schleswig-Holstein

Institute of Primary Medical Care

Arnold-Heller-Straße 3, Haus 9

25105 Kiel, Germany

kaduszkiewicz@allgemeinmedizin.uni-kiel.de

\begin{abstract}
PURPOSE The concept of mild cognitive impairment (MCI) has recently been introduced into the Diagnostic and Statistical Manual of Mental Disorders, Fifth Edition (DSM-5) as mild neurocognitive disorder, making it a formal diagnosis. We investigated the prognostic value of such a diagnosis and analyzed the determinants of the future course of $\mathrm{MCl}$ in the AgeCoDe study (German Study on Ageing, Cognition, and Dementia in Primary Care Patients).
\end{abstract}

METHODS We recruited 357 patients with $\mathrm{MCl}$ aged 75 years or older from primary care practices and conducted follow-up with interviews for 3 years. Depending on the course of impairment over time, the patients were retrospectively split into 4 groups representing remittent, fluctuating, stable, and progressive courses of $\mathrm{MCl}$. We performed ordinal logistic regression analysis and classification and regression tree (CART) analysis.

RESULTS Overall, $41.5 \%$ of the patients had remission of symptoms with normal cognitive function 1.5 and 3 years later, $21.3 \%$ showed a fluctuating course, $14.8 \%$ had stable symptoms, and $22.4 \%$ had progression to dementia. Patients were at higher risk for advancing from one course to the next along this spectrum if they had symptoms of depression, impairment in more than 1 cognitive domain, or more severe cognitive impairment, or were older. The result on a test of the ability to learn and reproduce new material 10 minutes later was the best indicator at baseline for differentiating between remittent and progressive $\mathrm{MCl}$. Symptoms of depression modified the prognosis.

CONCLUSIONS In primary care, about one-quarter of patients with $\mathrm{MCI}$ have progression to dementia within the next 3 years. Assessments of memory function and depressive symptoms are helpful in predicting a progressive vs a remittent course. When transferring the concept of $\mathrm{MCl}$ into clinical diagnostic algorithms (eg, DSM-5), however, we should not forget that three-quarters of patients with $\mathrm{MCl}$ stayed cognitively stable or even improved within 3 years. They should not be alarmed unnecessarily by receiving such a diagnosis.

Ann Fam Med 2014;158-165. doi:10.1370/afm.1596.

\section{INTRODUCTION}

M ild cognitive impairment $(\mathrm{MCI})$ is a common condition in the elderly with a prevalence of $16.0 \%$ in individuals without dementia $^{1}$ and an incidence rate of 63.6 (per 1,000 person-years). ${ }^{2}$ It is considered to be a transitional state between normal and pathologic cognitive decline. $\mathrm{MCI}$ is defined by a cognitive performance below that expected for age and educational attainment, but above a pathologic level as in early dementia. Patients show essentially normal functional activities.

Winblad et $\mathrm{al}^{3}$ classified the clinical presentations of $\mathrm{MCI}$ into subtypes according to the impaired cognitive domains, such as memory, orientation, intellectual abilities, and higher cortical functioning. Taking into account memory deficits and the number of cognitive domains impaired, MCI subtypes have been classified as follows: amnestic single domain (impairment of memory only), amnestic multidomain (impairment of memory and 1 or more other domains), nonamnestic multidomain (no memory impairment, 
but impairment of more than 1 of the other domains), and nonamnestic single domain (no memory impairment, but impairment of 1 of the other domains)., 4,5

Until now, MCI has been a concept mainly applied in research, however, $\mathrm{MCI}$ has recently been added as the diagnosis of mild neurocognitive disorder to the 5th edition of the Diagnostic and Statistical Manual of Mental Disorders (DSM-5). ${ }^{6}$ This transition from research to clinical practice aims at recognizing the substantial clinical needs of affected individuals. At the same time, it carries the risk of causing uncertainty and overtreatment of patients with MCI who may never have progression to dementia. When patients worried about their cognition consult their general practitioner, he/she will need more information on the prognostic value of such a diagnosis and on the determinants of its future course; however, research thus far has found $\mathrm{MCI}$ to be a condition with an unclear prognosis. ${ }^{7}$

$\mathrm{MCI}$ can take 4 courses, ranked by increasing severity: remittent (with recovery to normal cognitive function), fluctuating (changing between $\mathrm{MCI}$ and normal cognitive function), stable (impairment at each assessment that neither worsens to dementia nor improves to normal cognitive function), and progressive (development of dementia). So far, most studies have focused on the progressive course and its determinants.

In this study, AgeCoDe (German Study on Ageing, Cognition and Dementia in Primary Care Patients), we investigated determinants of the future course of MCI in primary care patients. We calculated a decision tree with factors that differentiate best between the $4 \mathrm{MCI}$ courses, which will help general practitioners counsel patients about prognosis. We focused on factors that are inexpensive and, in principle, accessible in general practice.

\section{METHODS}

Our study methods have been described elsewhere. ${ }^{8}$ In brief, we recruited 3,327 primary care patients in 6 German cities in 2003-2004. Inclusion criteria were an age of 75 years or older, absence of dementia, and at least 1 contact with the general practitioner within the last 12 months. Exclusion criteria were consultations only by home visits, residence in a nursing home, severe illness the practitioner would deem fatal within 3 months, insufficient facility in German, deafness, blindness, inability to consent, and not being a regular patient of the practice. The study was approved by the local ethics boards of all participating centers. Written informed consent was obtained from all patients.*

\footnotetext{
*The term patients is used because the participants of this study were recruited in primary care. It does not imply that the study participants were under medical treatment because of their mild cognitive impairment.
}

\section{Assessment Procedures}

Trained interviewers (physicians or psychologists) visited the patients at their homes and carried out assessments at baseline and 18 and 36 months later. Neuropsychological assessment was based on 4 instruments for the diagnosis of dementia: (1) the Structured Interview for Diagnosis of Dementia of Alzheimer type, Multi-infarct Dementia, and Dementia of other Aetiology according to the Diagnostic and Statistical Manual of Mental Disorders, 3rd Edition, Revised (DSM-III-R), Diagnostic and Statistical Manual of Mental Disorders, 4th Edition (DSM$I V)$, and International Classification of Diseases, 10 th Revision $\left(\right.$ ICD-10) $\left(\right.$ SIDAM $_{i}$ range $=0$ to 55$){ }_{1}^{9}(2)$ a screening test for cognitive impairment, the Mini-Mental State Examination (range $=0$ to 30 ), ${ }^{10}$ (3) the verbal fluency subtest (1 point for each named animal within $60 \mathrm{sec}-$ onds), word list memory subtest (range $=0$ to 30 ), word list delayed recall subtest (range $=0$ to 10 ), and word list recognition subtest (range $=0$ to 10 ) of the Neuropsychological battery of the Consortium to Establish a Registry for Alzheimer's Disease (CERAD), ${ }_{1}^{11}$ and (4) the clock-drawing test (range $=0$ to 10$),{ }^{12}$ a screening test for dementia. For all above cognitive scales, a higher score indicates a better cognitive performance.

We measured depression with the short version of the Geriatric Depression Scale (range $=0$ to 10 ; higher score indicates more severe depressive symptoms ${ }^{13}$; activities of daily living with the SIDAM activities of daily living scale (range $=0$ to 14 ; higher score indicates better performance $)^{9} ;$ and instrumental activities of daily living with the scale of Lawton and Brody (range $=0$ to 8 ; higher score indicates better performance). ${ }^{14}$ We classified education according to CASMIN criteria (Comparative Analysis of Social Mobility in Industrial Nations; categories = low, middle, high education). ${ }^{15}$ Additionally, patients were asked whether they had problems with mobility, vision, and hearing (categorized as yes or no for each). After each assessment, the interviewer rated the patient's severity of cognitive decline on the Global Deterioration Scale (GDS) of Reisberg et al (range $=1$ to 7 ; higher score indicates more severe impairment). ${ }^{16} \mathrm{~A}$ blood sample was taken to determine whether patients carried the apolipoprotein E $\varepsilon 4$ allele (ApoE $\varepsilon 4$ ).

\section{Definition of Cases}

We diagnosed MCI by applying consensus criteria proposed by the International Working Group on Mild Cognitive Impairment. ${ }^{3}$ These criteria have been shown to be superior to former $\mathrm{MCI}$ definitions for predicting dementia in general practice. ${ }^{17}$ They include (1) absence of dementia, (2) evidence of cognitive decline, from a self-rating or informant report and impairment on objective cognitive tasks and/or 
evidence of decline over time on objective cognitive tasks, and (3) preserved baseline activities of daily living and minimal impairment in complex instrumental functions. Dementia at baseline was excluded by an interviewer-rated GDS score of 4 or higher. Evidence of cognitive decline was defined as a SIDAM score more than 1 standard deviation below the age- and education-specific norm ${ }^{8}$ in 1 of the 4 cognitive domains it assesses (memory, orientation, intellectual abilities, and higher cortical functioning).

We categorized the patients into the $4 \mathrm{MCI}$ subtypes based on their objective cognitive impairment in the cognitive domains measured by the SIDAM. Individuals with impairment in only the memory domain were classified as having amnestic single-domain MCI. Nonamnestic single-domain $\mathrm{MCI}$ was diagnosed only if a single domain other than memory was impaired. If at least 2 domains other than memory showed objective impairment, patients received a diagnosis of nonamnestic multidomain MCI. Amnestic multidomain MCI was diagnosed if memory and at least 1 other domain were impaired.

Dementia was considered in a patient at the first and second follow-ups based on SIDAM score, an interviewer-rated GDS of at least 4, or both. A definitive diagnosis of dementia was made in a consensus conference with the interviewer and an experienced geriatrician or geriatric psychiatrist according to the set of criteria in the DSM-IV, which are implemented as a diagnostic algorithm in the SIDAM, and taking into account all other information documented. We included all patients with an MCI diagnosis at baseline who had complete SIDAM scores at each assessment or a diagnosis of dementia at any follow-up (Figure 1).

After follow-up, all patients were allocated to 1 of the 4 possible courses of $\mathrm{MCI}$ : remittent, fluctuating, stable, or progressive. The approach used to define these courses is shown in Figure 2.

\section{Data Analysis}

We performed statistical analyses with SPSS for Windows, versions 15 and 16 (SPSS Inc). All possible determinants of courses derived at baseline were analyzed in bivariate analyses and multivariate stepwise ordinal logistic regression analyses with the 4 possible courses of MCI as the target variable. Additionally, we performed classification and regression tree (CART) analysis to identify predictors that can discriminate between the courses of MCI. The CART method is based on recursive partitioning analysis ${ }^{18}$; the aim is to form prediction rules by constructing binary trees. Splitting rules are used as criteria to select the best split at each node; in this analysis, we used the Gini index of diversity as a measure of node Impurity as a

\section{Figure 1. Sampling frame and sample.}

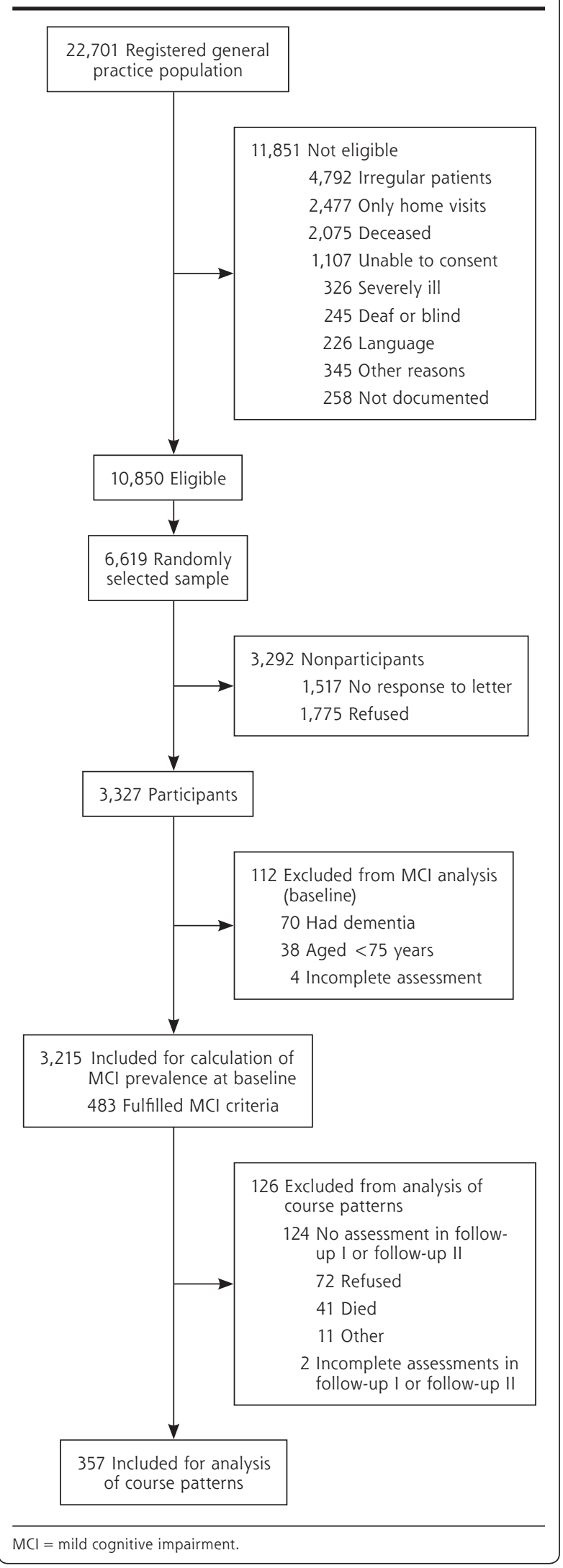


Figure 2. Definitions and prevalences of $\mathrm{MCl}$ courses.

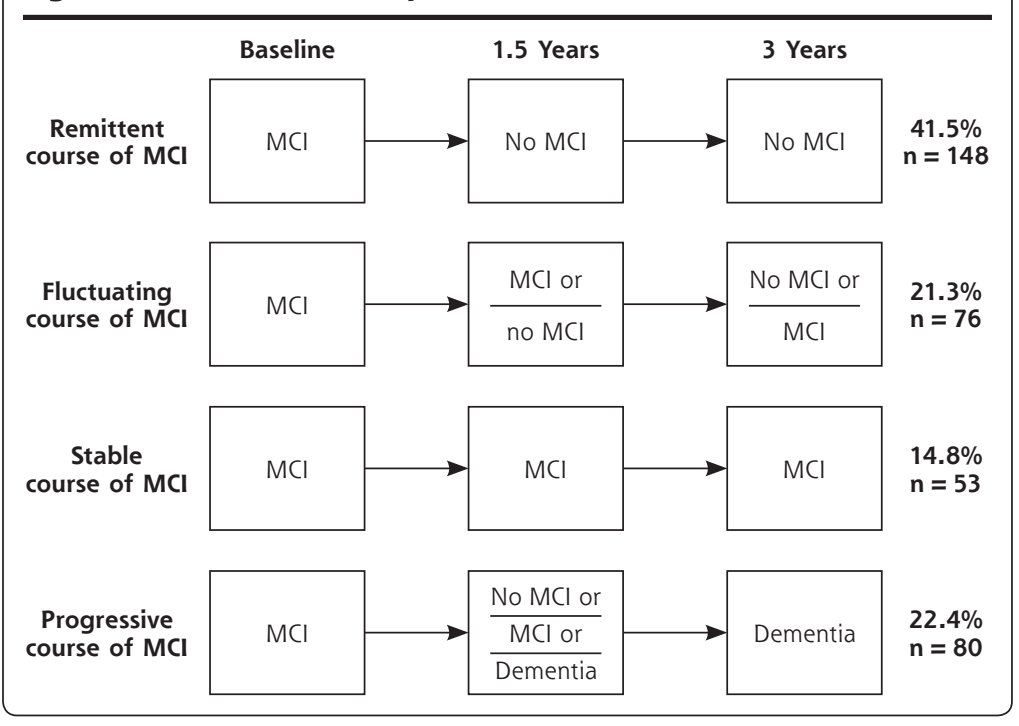

and reduced ability to take responsibility for medication and finances (for all $P<.001)$, as well as ApoE4 $\varepsilon 4$ carrier status $(P=.001)$ and reduced ability to use the telephone $(P=.002)$. There was also a significant although slightly weaker association with older age $(P=.007)$. Sex, education, family history of dementia, vision, and hearing ability were not significantly associated with the course of $\mathrm{MCI}$.

We next considered the 4 courses of $\mathrm{MCI}$ as an ordinal scale of increasing severity: remittent, fluctuating, stable, and progressive. Table 2 shows the results of the multivariate stepwise ordinal logistic regression analysis with the 4 courses of $\mathrm{MCI}$ ranked from least severe (remittent) to the most severe (progressive) defined as outcome vari-

splitting rule. A 10 -fold cross-validation was used to accurately assess its goodness of fit. CART analysis has several advantages over traditional methods, including logistic regression models. It is nonparametric; no assumptions are made regarding the underlying distribution of values of the discriminator with respect to predictor variables. It can handle numerical data that are highly skewed or multimodal, including categorical predictors. CART is often able to uncover complex interactions or patterns between predictors that may be difficult or impossible to uncover using traditional multivariate techniques. CART also produces trees that are relatively simple for nonstatisticians to interpret.

\section{RESULTS}

The characteristics of the patients at baseline are given in Table 1 . We excluded from analyses $126(26 \%)$ of 483 patients at baseline because they dropped out or had insufficient data to determine the presence of MCI.

The distribution of the patients according to the 4 courses of MCI during a mean \pm standard deviation follow-up time of $2.94 \pm 0.44$ years is displayed in Figure 2 .

\section{Determinants of the Courses of $\mathrm{MCl}$}

In bivariate analyses, variables significantly associated with an increasingly severe course of MCI were the subtype (predominantly the multidomain amnestic subtype); more severe cognitive impairment detected by 4 CERAD subtests (verbal fluency, word list memory, word list delayed recall, and word list recognition) and by the clock drawing test ${ }_{i}$ more depressive symptoms; reduced ability to walk; reduced SIDAM activities of daily living score; reduced ability to use transportation; able. Predictors of an increasingly severe course of $\mathrm{MCI}$ were worse performance in delayed recall tasks (CERAD subtest word list delayed recall) and worse ability to learn new material (CERAD subtest word list

Table 1. Patient Characteristics

\begin{tabular}{|c|c|c|c|}
\hline Characteristic & $\begin{array}{l}\text { Included } \\
\text { Patients }\end{array}$ & $\begin{array}{l}\text { Excluded } \\
\text { Patients }\end{array}$ & $\begin{array}{c}P \\
\text { Value }\end{array}$ \\
\hline Number & 357 & 126 & \\
\hline Age, mean (SD), y & $79.9(3.8)$ & $80.4(4.1)$ & .24 \\
\hline Female sex, No. (\%) & $234(65.5)$ & $88(69.8)$ & .38 \\
\hline Education level (CASMIN), \% & & & .02 \\
\hline Low & 40.1 & 51.6 & \\
\hline Medium & 44.3 & 38.9 & \\
\hline High & 15.7 & 9.5 & \\
\hline SIDAM score, mean (SD) & $44.8(5.2)$ & $43.3(4.9)$ & .006 \\
\hline $\begin{array}{l}\text { Global Deterioration Scale } \\
\text { cognitive impairment, \% }\end{array}$ & & & .18 \\
\hline No & 24.4 & 16.7 & \\
\hline Questionable & 29.4 & 30.2 & \\
\hline Little & 46.2 & 53.2 & \\
\hline $\begin{array}{l}\text { Mini-Mental State Examina- } \\
\text { tion score, mean (SD) }\end{array}$ & $26.1(2.2)$ & $25.8(2.1)$ & .25 \\
\hline ApoE $\varepsilon 4$ carrier, $\%$ & 27.1 & 20.3 & .14 \\
\hline $\begin{array}{l}\text { MCl subtype by Winblad } \\
\text { et } \mathrm{al}^{3} \text {, No. (\%) }\end{array}$ & & & .18 \\
\hline Nonamnestic single domain & $217(60.8)$ & $64(50.8)$ & \\
\hline Amnestic single domain & $46(12.9)$ & $19(15.1)$ & \\
\hline Nonamnestic multidomain & $38(10.6)$ & $21(16.7)$ & \\
\hline Amnestic multidomain & $56(15.7)$ & $22(17.5)$ & \\
\hline \multicolumn{4}{|c|}{$\begin{array}{l}\text { ApoE } \varepsilon 4=\text { apolipoprotein E } \varepsilon 4 \text { allele; CASMIN = Comparative Analysis of } \\
\text { Social Mobility in Industrial Nations; } \mathrm{MCI}=\text { mild cognitive impairment; } \\
\text { SIDAM = Structured Interview for Diagnosis of Dementia of Alzheimer type, } \\
\text { Multi-infarct Dementia, and Dementia of other Aetiology according to the } \\
\text { Diagnostic and Statistical Manual of Mental Disorders, 3rd Edition, Revised (DSM- } \\
\text { III-R), Diagnostic and Statistical Manual of Mental Disorders, 4th Edition (DSM-IV), } \\
\text { and International Classification of Diseases, 10th Revision (ICD-10). }\end{array}$} \\
\hline
\end{tabular}


trate that patients with $\mathrm{MCI}$ are a heterogeneous group with respect to disease course: $41.5 \%$ had a remittent course, $21.3 \%$ a fluctuating course, $14.8 \%$ a stable course, and $22.4 \%$ a progressive course. In comparing this study with other prospective studies of $\mathrm{MCI}$, we have selected only those studies that applied the MCI criteria proposed by the International Working Group ${ }^{3}$ and were performed in primary care or population-based settings.

\section{Progressive Course}

Conversion rates from $\mathrm{MCI}$ to dementia have been investigated in studies with observation times of 1 to 6 years. ${ }^{19-23}$ The overall rates in those studies varied between $0.7 \%{ }^{19}$ and $51.6 \% .{ }^{23}$ In the majority of studies, patients with multidomain $\mathrm{MCl}$ were at higher risk for progression than patients with single-domain subtypes, ${ }^{20,24-26}$ but results vary regarding the exact rank order of risk associated with the subtypes. ${ }^{3}$ Furthermore, progression to dementia seems to occur primarily within the first months of observation with lower rates in later years. ${ }^{22,27}$

Our conversion rate of $22.4 \%$ in patients with a mean age of $79.9 \pm 3.8$ years lies between the $6.6 \%$ found in a considerably younger sample $(74.6 \pm 5.7 \text { years })^{21}$ and the $30 \%$ found in an older sample $(82.2 \pm 5.0$ years $){ }^{22}$ This pattern underlines earlier findings of an increased risk for progression to dementia in older age. ${ }^{21,26,28}$

\section{Remittent Course}

In other studies, the rate of remittent MCI has ranged from an annual rate of $12.3 \%$ within 3.8 years to a total rate of $28.6 \%$ within 2 years. ${ }^{19,20,23,25,29}$ The highest rates of remission have been associated with nonamnestic single-domain MCI. ${ }^{23}$ The $41.5 \%$ of patients having a remittent course in our study is consistent with the data of studies having similar follow-up periods, namely, $37 \%$ within 4 years $^{21}$ and an annual rate of $12.3 \%$ within 3.8 years. $^{29}$

\section{Stable Course}

The stable course of MCI has been less investigated; estimated rates range from $80 \%$ in 1 year to $0 \%$ to $11 \%$ after 5 years of observation. ${ }^{19,20,23,25}$ Two studies considering subtype found conflicting results based on small numbers of patients analyzed. ${ }^{23,25}$ The rates are inconsistent, even if observation time is considered. Studies with shorter follow-up have found higher rates of stable MCI. In our study, $14.8 \%$ of patients had a stable course. Comparability with these other studies is limited, as their definitions of stable differed from ours in the number of follow-ups and observation period.

\section{Fluctuating Course}

So far, only a single other study has investigated the fluctuating course of $\mathrm{MCI}$, finding that $14.1 \%$ of patients had this course within 1.5 to 6 years of followup, with the highest percentage among patients with nonamnestic multidomain $\mathrm{MCI} .{ }^{23}$ In our sample, $21.3 \%$ of patients had fluctuating MCI. The lower percentage found in the aforementioned study might reflect a more progression to dementia with longer observation. Additionally, their sample included institutionalizedand hence sicker-persons. Another study did not differentiate between the stable and fluctuating course of MCI and reported that $56.5 \%$ of patients continued to meet diagnostic criteria for MCI. ${ }^{21}$ This value is higher than that for our combined group of stable and fluctuating course $(36.1 \%)$, probably due to the younger age of that sample $(74.6 \pm 5.7$ years $)$.

\section{Prognosis of $\mathrm{MCl}$}

In our study, the MCI subtypes proposed by Winblad et $\mathrm{al}^{3}$ were associated with the future course of $\mathrm{MCI}$. Compared with the single-domain nonamnestic subtype, patients with multidomain amnestic MCI had a 3 times higher risk for a more severe course of $\mathrm{MCI}$, followed by patients with multidomain nonamnestic $\mathrm{MCI}$, whose risk was 2 times higher. Patients with the singledomain amnestic subtype did not have a significantly elevated risk. The increased risk of a more severe course among patients with the multidomain subtype supports earlier findings. ${ }^{20,24-26}$

Our CART analysis revealed that MCI subtype is not the best factor to predict the course of $\mathrm{MCI}$, however. The ability to learn new material, as measured by the CERAD word list memory subtest, best differentiated between remittent and progressive $\mathrm{MCI}$. This finding supports earlier findings that more severely impaired cognition predicts a progressive course ${ }^{28,30}$ and further reinforces the recommendations from the National Institute on Aging and the Alzheimer's Association work group to use word-list learning tests for cognitive assessment. ${ }^{31}$ Further, CART analyses showed that because of strong correlations, the ability to learn new material (CERAD word list memory) is a surrogate for the performance in delayed recall tasks (CERAD word list recall).

Predictors of stable, fluctuating, or remittent courses of MCI have not been well investigated. Besides the MCI subtype, fewer chronic medical conditions and younger age predict reversion to normal cognition. ${ }^{32}$ The only factors associated with fluctuating or stable MCI so far investigated are the different MCI subtypes, as discussed above. We investigated determinants for a more severe course of MCI considering the 4 courses ranked in order of severity. We found that symptoms of depression elevate the risk of a more severe course of $\mathrm{MCI}_{\text {; }}$ however, the cutoff score of 3.5 on the Geriatric Depression Scale found in the CART analysis lies within the range regarded as absence of depression ${ }^{33}$ and is 
clinically hardly detectable. Nonetheless, this shows that subdepressive symptoms are associated with a progressive course of $\mathrm{MCI}$. These findings concur with those of some studies ${ }_{1}^{21,29}$ whereas another study did not find this association in patients with $\mathrm{MCI}$, but only in patients without MCI who had progression to dementia. ${ }^{34}$ Some investigators have suggested that depression may be an early manifestation rather than a risk factor for dementia or that depression unmasks $\mathrm{MCI}$ in patients with limited cognitive reserve, but is not a symptom of the neurologic condition that causes dementia. ${ }^{35}$ Age significantly influenced the course of $\mathrm{MCI}$, but was not important enough to play a role in the CART analysis.

\section{MCI in Clinical Practice}

The value of diagnosing mild neurocognitive disorder, as in the DSM-5, especially in general practice, remains questionable. Patients given this diagnosis will be a heterogeneous group, and it will not have any immediate consequences in the large majority of cases. Even if there were preventive treatments, many patients would be treated unnecessarily. If the diagnosis of $\mathrm{MCI}$ is transferred into clinical practice within primary care, diagnostic criteria should be specified and focused on the subgroup of patients at high risk for progressive $\mathrm{MCI}$. We believe that in the long term, primary care clinicians will be affected by the DSM-5 as many patients return to their general practitioner for care after a specialist diagnosis.

\section{Strength and Limitations}

This is the first study to investigate determinants of the $4 \mathrm{MCI}$ courses. MCI diagnosis was based on the consensus criteria of the International Working Group, ${ }^{3}$ which have been validated within general practice. ${ }^{17}$ These criteria are very similar to the diagnostic criteria for mild neurocognitive disorder in the DSM-5. ${ }^{36}$

Limitations of our study relate to our focus on prevalent MCI. Rates for the different courses may vary depending on the time since onset. We found that $21.3 \%$ of patients switch between MCI and normal cognitive status; thus, the onset of $\mathrm{MCI}$ is not clearly defined. We therefore decided to investigate prevalent MCI. Because of the exclusion criteria, our study sample is representative of general practice patients who live at home and still are able to consult their practitioners in their office (a selection bias). A further limitation relates to the exclusion of some patients from analyses by attrition, mainly because of refusal and death. Compared with the included patients, the excluded patients had significantly lower SIDAM scores $(43.3 \pm 4.9$ vs $44.8 \pm 5.2)$ and a lower level of education. Both the selection bias and the attrition bias might have led to an overestimation of the proportion of patients with a remittent course and an underestimation of the proportion with a progressive course of $\mathrm{MCI}$.

\section{Implications}

In primary care, about one-quarter of patients with $\mathrm{MCI}$ experience progression to dementia within the next 3 years. The performance on tasks of learning new material (CERAD subtest word list memory) and the Geriatric Depression Scale, which can detect subdepressive symptomatology, help predict the progressive versus the remittent course of MCI. Patients at high risk for progression to dementia should be monitored regularly by the general practitioner. When transferring the concept of $\mathrm{MCI}$ into clinical diagnostic algorithms (eg, DSM-5), however, we should not forget that threequarters of patients with MCI stay cognitively stable or even improve within 3 years. Of those, one subgroup can be characterized well: patients with a CERAD word list memory score of at least 20 points and Geriatric Depression Scale score of 3 or less; $75 \%$ of these patients will return to normal cognitive function within the next 3 years. They should not be alarmed by receiving a clinical diagnosis of mild neurocognitive disorder.

To read or post commentaries in response to this article, see it online at http://www.annfammed.org/content/12/2/158.

Key words: mild cognitive impairment; primary care; dementia; Alzheimer disease; disease course; prognosis; practice-based research

Submitted December 5, 2012; submitted, revised, May 23, 2013; accepted July 26, 2013.

Author affiliations: Department of Primary Medical Care, Center for Psychosocial Medicine, University Medical Center, Hamburg-Eppendorf, Germany (Kaduszkiewicz, Eisele, van den Bussche, Scherer); Hannover Medical School, Hannover, Germany (Wiese, Prokein); Institute of Primary Medical Care, University Medical Center Schleswig-Holstein (Kaduszkiewicz); Institute of Social Medicine, Occupational Health and Public Health, University of Leipzig, Germany (Luppa, Luck, Riedel-Heller); Department of Psychiatry, University of Bonn, Germany (Jessen, Maier); Department of Psychiatry, Technical University of Munich, Germany (Bickel, Mösch); Institute of General Practice, Medical Faculty, Heinrich-Heine-University Düsseldorf, Germany (Pentzek, Fuchs); Central Institute of Mental Health, Medical Faculty Mannheim/Heidelberg University, Mannheim, Germany (Eifflaender-Gorfer, Weyerer); Department of Health Economics and Health Services Research, University Medical Center Hamburg-Eppendorf, Germany (König, Brettschneider); German Center for Neurodegenerative Diseases (DZNE), Bonn, Germany (Maier, Jessen).

Funding support: This study and publication are part of the German Research Network on Dementia (KND) and the German Research Network on Degenerative Dementia (KNDD) and were funded by the German Federal Ministry of Education and Research (grants KND: 01GI0102, 01GI0420, 01GI0422, 01GI0423, 01GI0429, 01GI0431, 01GI0433, 01GI0434; grants KNDD: 01GI0710, 01GI0711, 01GI0712, 01GI0713, 01GI0714, 01GI0715, 01GI0716).

Previous presentations: Alzheimer's Association 2009 International Conference on Alzheimer's Disease (ICAD), July 11-16, 2009; Vienna, Austria. World Congress of the International Association of Gerontology 
and Geriatrics (IAGG), July 5- 9, 2009; Paris, France. Congress of the German Association for Psychiatry and Psychotherapy (DGPPN), November 23-26. 2011; Berlin, Germany.

Acknowledgments: We thank all participating patients and their general practitioners for their good collaboration.

Members of the AgeCoDe Study Group: Wolfgang Maier and Martin Scherer (principal investigators), Hendrik van den Bussche (principal investigator 2002-2011), Heinz-Harald Abholz, Christian Brettschneider, Cadja Bachmann, Horst Bickel, Wolfgang Blank, Sandra EifflaenderGorfer, Marion Eisele, Annette Ernst, Angela Fuchs, Kathrin Heser, Frank Jessen, Hanna Kaduszkiewicz, Teresa Kaufeler, Mirjam Köhler, HansHelmut König, Alexander Koppara, Carolin Lange, Tobias Luck, Melanie Luppa, Manfred Mayer, Edelgard Mösch, Julia Olbrich, Michael Pentzek, Jana Prokein, Anna Schumacher, Steffi Riedel-Heller, Janine Stein, Susanne Steinmann, Franziska Tebarth, Michael Wagner, Klaus Weckbecker, Dagmar Weeg, Jochen Werle, Siegfried Weyerer, Birgitt Wiese, Steffen Wolfsgruber, Thomas Zimmermann.

\section{References}

1. Petersen RC, Roberts RO, Knopman DS, et al; The Mayo Clinic Study of Aging. Prevalence of mild cognitive impairment is higher in men. Neurology. 2010;75(10):889-897.

2. Roberts RO, Geda YE, Knopman DS, et al. The incidence of $\mathrm{MCl}$ differs by subtype and is higher in men: the Mayo Clinic Study of Aging. Neurology. 2012;78(5):342-351.

3. Winblad $B$, Palmer $K$, Kivipelto $M$, et al. Mild cognitive impairment-beyond controversies, towards a consensus: report of the International Working Group on Mild Cognitive Impairment. J Intern Med. 2004;256(3):240-246.

4. Petersen RCDR, Doody R, Kurz A, et al. Current concepts in mild cognitive impairment. Arch Neurol. 2001;58(12):1985-1992.

5. Petersen RC, Smith GE, Waring SC, Ivnik RJ, Tangalos EG, Kokmen E. Mild cognitive impairment: clinical characterization and outcome. Arch Neurol. 1999;56(3):303-308.

6. American Psychiatric Association. DSM-5: The Future of Psychiatric Diagnosis. 2001. http://www.dsm5.org/Pages/Default.aspx. Accessed Apr 30, 2012.

7. Visser PJ, Brodaty H. MCI is not a clinically useful concept. Int Psychogeriatr. 2006;18(3):402-409, discussion 409-414.

8. Luck T, Riedel-Heller SG, Kaduszkiewicz H, et al; AgeCoDe group. Mild cognitive impairment in general practice: age-specific prevalence and correlate results from the German Study on Ageing, Cognition and Dementia in Primary Care Patients (AgeCoDe). Dement Geriatr Cogn Disord. 2007;24(4):307-316.

9. Zaudig M, Mittelhammer J, Hiller W, et al. SIDAM-a structured interview for the diagnosis of dementia of the Alzheimer type, multi-infarct dementia and dementias of other aetiology according to ICD-10 and DSM-III-R. Psychol Med. 1991;21(1):225-236.

10. Folstein MF, Folstein SE, McHugh PR. "Mini-mental state." A practical method for grading the cognitive state of patients for the clinician. J Psychiatr Res. 1975;12(3):189-198.

11. Morris JC, Heyman A, Mohs RC, et al. The Consortium to Establish a Registry for Alzheimer's Disease (CERAD). Part I. Clinical and neuropsychological assessment of Alzheimer's disease. Neurology. 1989;39(9):1159-1165.

12. Ihl R, Grass-Kapanke B. Test zur Früherkennung von Demenzen. Germany: Books on Demand; 2000.

13. Yesavage JA, Sheikh JI. Geriatric Depression Scale (GDS): recent evidence and development of a shorter version. Clin Gerontol. 1986; 5(1/2):165-173.

14. Lawton MP, Brody EM. Assessment of older people: self-maintaining and instrumental activities of daily living. Gerontologist. 1969; 9(3):179-186.
15. Brauns H, Steinmann S. Educational reform in France, West-Germany and the United Kingdom. ZUMA-Nachrichten. 1999;44(23):7-44.

16. Reisberg B, Ferris SH, de Leon MJ, Crook T. The Global Deterioration Scale for assessment of primary degenerative dementia. Am J Psychiatry. 1982;139(9):1136-1139.

17. Artero S, Petersen R, Touchon J, Ritchie K. Revised criteria for mild cognitive impairment: validation within a longitudinal population study. Dement Geriatr Cogn Disord. 2006;22(5-6):465-470.

18. Breiman L, Friedman J, Olshen R, Stone C. Classification and Regression Trees. Belmont, CA: Wadsworth; 1984.

19. Ganguli M, Snitz BE, Saxton JA, et al. Outcomes of mild cognitive impairment by definition: a population study. Arch Neurol. 2011; 68(6):761-767.

20. Brodaty $\mathrm{H}$, Heffernan $M$, Kochan $N$, et al. Incidence, prevalence and predictors of course of mild cognitive impairment: The Sydney Memory and Ageing Study. Alzheimers Dement. 2011;7(4):S535.

21. Artero $S$, Ancelin M-L, Portet F, et al. Risk profiles for mild cognitive impairment and progression to dementia are gender specific. J Neurol Neurosurg Psychiatry. 2008;79(9):979-984.

22. Busse A, Angermeyer MC, Riedel-Heller SG. Progression of mild cognitive impairment to dementia: a challenge to current thinking. Br J Psychiatry. 2006;189(5):399-404.

23. Busse A, Hensel A, Gühne U, Angermeyer MC, Riedel-Heller SG. Mild cognitive impairment: long-term course of four clinical subtypes. Neurology. 2006;67(12):2176-2185.

24. Palmer K, Bäckman L, Winblad B, Fratiglioni L. Mild cognitive impairment in the general population: occurrence and progression to Alzheimer disease. Am J Geriatr Psychiatry. 2008;16(7):603-611.

25. Ritchie LJ, Tuokko H. Patterns of cognitive decline, conversion rates, and predictive validity for 3 models of $\mathrm{MCl}$. Am J Alzheimers Dis Other Demen. 2010;25(7):592-603.

26. Baars MAE, van Boxtel MPJ, Dijkstra JB, et al. Predictive value of mild cognitive impairment for dementia. The influence of case definition and age. Dement Geriatr Cogn Disord. 2009;27(2):173-181.

27. Mitchell AJ, Shiri-Feshki M. Temporal trends in the long term risk of progression of mild cognitive impairment: a pooled analysis. J Neurol Neurosurg Psychiatry. 2008;79(12):1386-1391.

28. Amieva $\mathrm{H}$, Letenneur L, Dartigues J-F, et al. Annual rate and predictors of conversion to dementia in subjects presenting mild cognitive impairment criteria defined according to a population-based study. Dement Geriatr Cogn Disord. 2004;18(1):87-93.

29. Roberts R, Knopman D, Boeve B, et al. Outcomes of MCl: The Mayo Clinic study of aging. Alzheimers Dement. 2011;7(4):S551.

30. Ritchie K, Artero S, Touchon J. Classification criteria for mild cognitive impairment: a population-based validation study. Neurology. 2001;56(1):37-42.

31. Albert MS, DeKosky ST, Dickson D, et al. The diagnosis of mild cognitive impairment due to Alzheimer's disease: recommendations from the National Institute on Aging-Alzheimer's Association workgroups on diagnostic guidelines for Alzheimer's disease. Alzheimers Dement. 2011;7(3):270-279.

32. Olazarán I, Torrero $\mathrm{P}$, Cruz I, et al. Mild cognitive impairment and dementia in primary care: the value of medical history. Fam Pract. 2011;28(4):385-392.

33. Gauggel S, Birkner B. Validity and reliability of a German version of the Geriatric Depression Scale (GDS). [In German.] Z Klin Psychol. 1999;28(1):18-27.

34. Palmer K, Berger AK, Monastero R, Winblad B, Bäckman L, Fratiglioni L. Predictors of progression from mild cognitive impairment to Alzheimer disease. Neurology. 2007;68(19):1596-1602.

35. Panza F, Frisardi V, Capurso C, et al. Late-life depression, mild cognitive impairment, and dementia: possible continuum? Am J Geriatr Psychiatry. 2010;18(2):98-116.

36. American Psychiatric Association. Diagnostic and Statistical Manual of Mental Disorders, Fifth Edition. DSM-5. 5. Washington, DC: American Psychiatric Publishing; 2013. 